\title{
MicroRNA-219 exerts a tumor suppressive role in glioma via targeting Sal-like protein 4
}

\author{
BOTAO JIANG $^{1 *}$, MIN LI $^{2}$, FANG JI $^{3}$ and YAXIONG NIE ${ }^{4}$ \\ ${ }^{1}$ Department of Neurology, First Hospital of Changsha, Changsha, Hunan 430100; ${ }^{2}$ Department of Nephrology; ${ }^{3}$ Teaching \\ and Research Office of Medical Imaging, Third Xiangya Hospital of Central South University, Changsha, Hunan 410013; \\ ${ }^{4}$ Department of Neurology, First Affiliated Hospital of Nanhua University, Hengyang, Hunan 421001, P.R. China
}

Received October 11, 2016; Accepted June 2, 2017

DOI: $10.3892 /$ etm.2017.5292

\begin{abstract}
MicroRNAs (miRs) serve important roles in the development and progression of various human cancer types, including glioma. Recently, miR-219 has been suggested to function as a tumor suppressor in glioma; however, the underlying mechanism remains largely unknown. The aim of this study was to investigate the regulatory mechanism of miR-219 in the malignant phenotypes of glioma cells. Quantitative polymerase chain reaction (qPCR) and western blotting were conducted to examine the mRNA and protein expression. An MTT assay, wound healing assay and Transwell assay were used to study cell proliferation, migration and invasion. The qPCR data indicated that the expression of miR-219 was significantly decreased in glioma tissues compared with normal brain tissues. In addition, a low expression of miR-219 was identified to be associated with an advanced pathological grade. In vitro experiments demonstrated that miR-219 was also downregulated in several common glioma cell lines, including A172, U87, U251 and U373, when compared with that in normal astrocytes. Ectopic expression of miR-219 caused a significant decrease in U87 cell proliferation, migration and invasion. Luciferase reporter assay data indicated that Sal-like protein 4 (SALL4) was a direct target gene of miR-219, while the protein expression of SALL4 was negatively regulated by miR-219 in U87 cells. Furthermore, SALL4 was significantly upregulated in glioma tissues and cell lines, and upregulation of SALL4 was associated with a higher pathological grade. Furthermore, overexpression of SALL4 significantly attenuated the suppressive effects of miR-219 on U87 cell proliferation, migration and invasion, suggesting that miR-219
\end{abstract}

Correspondence to: Professor Botao Jiang, Department of Neurology, First Hospital of Changsha, 311 Yingpan Road, Changsha, Hunan 430100, P.R. China

E-mail: jiangbotaojf@qq.com

*Contributed equally

Key words: glioma, microRNA-219, Sal-like protein 4, tumor suppressor serves a suppressive role in glioma growth and metastasis via targeting SALL4. Therefore, the present study highlighted the clinical significance of the miR-219/SALL4 axis in glioma.

\section{Introduction}

Glioma is the most common primary brain malignant tumor, accounting for $30 \%$ of central nervous system tumors and $80 \%$ of all malignant tumors in the brain $(1,2)$. Despite great improvements in the diagnosis and treatment of this tumor, the prognosis of glioma patients at a late stage remains poor $(1,2)$. Deregulations of oncogenes or tumor suppressors have been identified in glioma in the past decade (3). Therefore, understanding the molecular mechanism underlying glioma progression is beneficial for the development of novel diagnostic and therapeutic strategies for this disease.

MicroRNAs (miRs), a class of non-coding RNAs with a length of 18-25 nucleotides, function as important regulators of gene expression by binding to the complementary regions of their target mRNAs, leading to protein translation inhibition or mRNA degradation $(4,5)$. Through inhibition of their target genes, miRs serve key roles in a variety of cellular biological processes, including cell survival, proliferation, cell cycle progression, differentiation, apoptosis, migration, as well as tumorigenesis (5-7). In recent years, numerous miRs have been reported to function as oncogenes or tumor suppressors in glioma $(8,9)$. For instance, miR-30a-5p promotes glioma cell growth by targeting septin 7 (9), while miR-124 inhibits the migration and invasion of glioma cells by directly inhibiting the expression of Rho-associated protein kinase 1 (8). However, the molecular mechanism underlying the role of miRs in glioma progression remains largely unclear.

miR-219 has been demonstrated to generally serve a tumor suppressive role in human cancer. For instance, Lei et al (10) demonstrated that miR-219 played anti-proliferative, pro-apoptotic and anti-metastatic roles, as well as reduced the levels of phosphorylated extracellular signal-regulated kinases $1 / 2$ in gastric cancer cells. In addition, Huang et al (11) reported that miR-219 inhibited hepatocellular carcinoma cell proliferation by targeting glypican-3. Recently, miR-219-5p has been identified to exert a tumor suppressor function by targeting roundabout guidance receptor 1 and epidermal growth factor receptor (EGFR) in glioblastoma, the most malignant type of 
glioma $(12,13)$. However, certain other target genes of miR-219 may also serve key roles in glioma, and the regulatory mechanism of miR-219 underlying glioma growth and metastasis needs to be fully uncovered.

Therefore, the present study aimed to investigate the clinical significance of miR-219 expression in glioma through the examination of glioma tissues and various glioma cell lines. Furthermore, the molecular mechanism of miR-219 underlying glioma malignant progression was investigated.

\section{Materials and methods}

Clinical tissue samples. The study was approved by the Ethics Committee of the First Hospital of Changsha (Changsha, China). A total of 63 glioma tissues and 12 normal brain tissues were obtained from the hospital between July 2010 and March 2012. The tissues were resected during surgery. Written informed consents were obtained from all patients. The clinical information of glioma patients included in the current study is summarized in Table I. The glioma patients were diagnosed according to the World Health Organization (WHO) stage and grading system (14). The Karnofsky performance scale (KPS) runs from 100 to 0 , where 100 is 'perfect health' and 0 is death. Its purpose is to allow physicians to evaluate a patient's ability to survive chemotherapy for cancer (15). All patients involved in this study received no treatment prior to surgical resection. All tissue samples were immediately snap-frozen and stored in liquid nitrogen until further use.

Cell culture. Normal human astrocytes were purchased from the IBS Cell Bank of Fudan University (Shanghai, China), and cultured in astrocyte media (ScienCell Research Laboratories, Inc., Carlsbad, CA, USA) with 10\% fetal bovine serum (FBS; Thermo Fisher Scientific, Inc., Waltham, MA, USA) at $37^{\circ} \mathrm{C}$ in a humidified incubator containing $5 \% \mathrm{CO}_{2}$. In addition, human glioma cell lines, including A172, U87, U251 and U373, were purchased from the Cell Bank of Chinese Academy of Sciences (Shanghai, China). Cells were cultured in Dulbecco's modified Eagle's medium (DMEM; Thermo Fisher Scientific, Inc.) supplemented with $10 \% \mathrm{FBS}$ in a $37^{\circ} \mathrm{C}$ humidified atmosphere with $5 \% \mathrm{CO}_{2}$.

Cell transfection. Cell transfection was conducted in the U87 glioma cell lines using Lipofectamine 2000 (Thermo Fisher Scientific, Inc.), according to the manufacturer's instructions. Briefly, U87 cells were cultured to $70 \%$ confluence, and resuspended in serum-free DMEM. Scramble miR (miR-NC), miR-219 mimic for miR-219 upregulation, negative control (NC) inhibitor, miR-219 inhibitor for miR-219 knockdown, and pc-DNA3.1-Sal-like protein 4 (SALL4) open reading frame plasmid for SALL4 upregulation were diluted in OPTI-MEM (Thermo Fisher Scientific, Inc.), which was then added with diluted Lipofectamine 2000. All mimics, inhibitors and plasmids were purchased from Yearthbio (Changsha, China). Following incubation for $20 \mathrm{~min}$ at room temperature, the mixture was added into the $\mathrm{U} 87$ cell suspension. Following incubation at $37^{\circ} \mathrm{C}$ and $5 \% \mathrm{CO}_{2}$ for $6 \mathrm{~h}$, the transfection mixture was replaced with DMEM with $10 \%$ FBS. MiR-NC was the corresponding control for miR-219 mimic, and NC inhibitor was the corresponding control for miR-219 inhibitor.
Reverse transcription-quantitative polymerase chain reaction (RT-qPCR) analysis. Total RNA was extracted from tissues and cell lines using TRIzol reagent (Thermo Fisher Scientific, Inc.). RNA quality and concentration was measured using a Nanodrop 2000 spectrophotometer (Thermo Fisher Scientific, Inc.) RNA was converted into cDNA using PrimeScript 1st Strand cDNA Synthesis kit (Takara Bio, Inc., Tokyo, Japan). For miR-219 expression detection, the miRNA Q-PCR Detection kit (GeneCopoeia, Rockville, MD, USA) was used to conduct qPCR on an ABI 7300 Plus thermal cycler (Applied Biosystems; Thermo Fisher Scientific, Inc.). U6 gene was used as an internal control. For mRNA expression detection, SYBR Green I Real-Time PCR kit (Biomics Biopharma, Nantong, China) was used to conduct qPCR on an ABI 7300 Plus system. The primers for SALL4 were: Forward, 5'-AGCACA TCAACTCGGAGGAG-3' and reverse, 5'-CATTCCCTGGGT GGTTCACTG-3'. The primers for the internal control gene GADPH were: Forward, 5'-CTGGGCTACACTGAGCACC-3' and reverse, 5'-AAGTGGTCGTTGAGGGCAATG-3'. The reaction conditions were as follows: $95^{\circ} \mathrm{C}$ for $5 \mathrm{~min}$, followed by 40 cycles of $95^{\circ} \mathrm{C}$ for $15 \mathrm{sec}$ and $60^{\circ} \mathrm{C}$ for $30 \mathrm{sec}$. The relative expression levels were analyzed by the $2^{-\Delta \Delta \mathrm{Cq}}$ method (16). According to the mean value of miR-219 levels that served as the cutoff value, glioma tissues were divided into the high and low miR-219 expression groups.

Western blotting. U87 cells were lysed in ice-cold buffer containing $0.5 \mathrm{~mol} / 1$ Tris- $\mathrm{HCl}, \mathrm{pH} 7.4,1.5 \mathrm{~mol} / \mathrm{l} \mathrm{NaCl}$, $2.5 \%$ deoxycholic acid, 10\% NP-40 and $10 \mathrm{mmol} / \mathrm{l}$ EDTA (Sigma-Aldrich; Merck, Darmstadt, Germany) with cocktail protease inhibitors (Thermo Fisher Scientific, Inc.). Cell lysates were then obtained by centrifugation at $16,000 \mathrm{x} \mathrm{g}$ for $20 \mathrm{~min}$ at $4^{\circ} \mathrm{C}$. Protein concentrations were determined by Bio-Rad Protein Assay kit (Bio-Rad Laboratories, Inc., Hercules, CA, USA), and $50 \mu \mathrm{g}$ protein was subjected to $12 \%$ SDS-PAGE, followed by immunoblotting onto a polyvinylidene difluoride membrane (Thermo Fisher Scientific, Inc.). The membrane was blocked for $1 \mathrm{~h}$ in phosphate-buffered saline with Tween 20 (PBST) containing 5\% nonfat dry milk (Yili, Beijing, China). Subsequently, the membrane was incubated at room temperature for $3 \mathrm{~h}$ with primary antibodies: Rabbit polyclonal to SALL4 antibody (1:50, ab29112, Abcam, Cambridge, MA, USA) and rabbit polyclonal to GAPDH antibody (1:50, ab9485, Abcam). The membrane was then washed three times with PBST, then immersed in PBST containing horseradish peroxidase-conjugated goat anti-rabbit secondary antibody $(1: 5,000$, ab7090, Abcam) at room temperature for $1 \mathrm{~h}$. After washing in PBST four times (10 min/wash), an enhanced chemiluminescence kit (Thermo Fisher Scientific, Inc.) was used to perform chemiluminescence detection. The relative protein expression was determined using Image $\mathbf{J}$ software v.1.48 (National Institutes of Health, Bethesda, MD, USA), represented as the density ratio vs. GAPDH.

Cell proliferation assay. Cell proliferation was measured by MTT assay. U87 cells in $200 \mu 1$ serum-free DMEM were plated into 96-well plates at a density of 5,000 cells per well. After cells were adhered, the medium was replaced with DMEM containing $10 \% \mathrm{FBS}$, and incubated at $37^{\circ} \mathrm{C}$ for $12,24,48$ and $72 \mathrm{~h}$, respectively. Next, $20 \mu \mathrm{l}$ MTT solution $(5 \mathrm{mg} / \mathrm{ml})$ was 
Table I. Association between miR-219 levels and clinicopathological characteristics of glioma patients.

\begin{tabular}{|c|c|c|c|c|}
\hline \multirow[b]{2}{*}{ Variables } & \multirow[b]{2}{*}{ Cases, $\mathrm{n}$} & \multicolumn{2}{|c|}{$\begin{array}{l}\text { miR-219 } \\
\text { expression }\end{array}$} & \multirow[b]{2}{*}{ P-value } \\
\hline & & $\begin{array}{c}\text { Low } \\
(n=36)\end{array}$ & $\begin{array}{l}\text { High } \\
(n=27)\end{array}$ & \\
\hline Age, years & & & & $>0.999$ \\
\hline$<50$ & 25 & 20 & 15 & \\
\hline$\geq 50$ & 38 & 16 & 12 & \\
\hline Sex & & & & 0.430 \\
\hline Male & 40 & 21 & 19 & \\
\hline Female & 23 & 15 & 8 & \\
\hline WHO grade & & & & 0.010 \\
\hline I-II & 27 & 10 & 17 & \\
\hline III-IV & 36 & 26 & 10 & \\
\hline KPS & & & & 0.004 \\
\hline$>90$ & 24 & 8 & 16 & \\
\hline$\leq 90$ & 39 & 28 & 11 & \\
\hline
\end{tabular}

miR, microRNA; WHO, World Health Organization; KPS, Karnofsky performance scale.

added into each well. Following incubation at $37^{\circ} \mathrm{C}$ for $4 \mathrm{~h}$, the MTT solution was replaced with $150 \mu$ l dimethyl sulfoxide to dissolve the tetrazolium crystals for $10 \mathrm{~min}$. Absorbance at $570 \mathrm{~nm}$ was detected with a microplate reader (Thermo Fisher Scientific, Inc.).

Cell migration assay. U87 cells were plated at $1 \times 10^{5}$ cells/well in 24-well plates with $1 \mathrm{ml}$ DMEM and incubated for $24 \mathrm{~h}$. Scratch wounds were generated using a $200 \mu$ l pipette tip. The medium was then replaced with fresh DMEM with $10 \%$ FBS. The wound size was recorded by camera at 0 and $24 \mathrm{~h}$, respectively. Quantification was implemented by Image $\mathrm{J}$ v1.48 software.

Cell invasion assay. In this assay, 24-well-plate-matched Bio-coat cell invasion chambers (BD Biosciences, Franklin Lakes, NJ, USA) were used to measure the invasion of glioma cells. Briefly, $1.0 \times 10^{5} \mathrm{U} 87$ cells were seeded in the upper transwell chamber in serum-free medium, with $500 \mu 1$ DMEM containing $10 \%$ FBS in the lower chamber. Following incubation at $37^{\circ} \mathrm{C}$ for $24 \mathrm{~h}$, the cells that did not migrate through the pores were carefully wiped out with a cotton-tipped swab. The cells that had migrated were then fixed in $90 \%$ alcohol, followed by staining with $0.1 \%$ crystal violet (Sigma-Aldrich; Merck). Subsequent to washing with PBS for three times, invading cells were observed and photographed using an inverted microscope (Olympus Corp., Tokyo, Japan).

Bioinformatics analysis. Bioinformatics analysis was performed to predict the potential target genes of miR-219 using TargetScan online software (version 7.1; www.targetscan .org), according to the manufacturer's instructions. SALL4 was shown as the putative target gene of miR-219, and this association was then confirmed using a luciferase reporter gene assay.

Luciferase reporter gene assay. Luciferase reporter gene assay was conducted to confirm the association between miR-219 and its potential target, SALL4. Initially, the mutant type (MT) of SALL4 3'-untranslated region (UTR) lacking complementarity with the miR-219 binding sequence was constructed using QuickChange Site-Directed Mutagenesis kit (Stratagene; Agilent Technologies, Inc., Santa Clara, CA, USA), according to the manufacturer's instructions. The wild type (WT) or mutant type (WT) 3'UTRs of SALL4 were also constructed and inserted into the multiple cloning sites in the psiCHECK2 luciferase reporter vector (Promega Corp., Madison, WI, USA). Lipofectamine 2000 was used to co-transfected U87 cells with WT-SALL4-3'UTR or MT-SALL4-3'UTR reporter plasmids, and miR-219 mimics or miR-NC. At $48 \mathrm{~h}$ after co-transfection, the luciferase activities were examined using a dual-luciferase reporter assay system (Promega Corp.), according to the manufacturer's protocol. The Renilla luciferase activity was normalized to the firefly luciferase activity.

Statistical analysis. Data in the present study are expressed as the mean \pm standard deviation of three independent experiments. The difference between two groups was analyzed using Student's t-test, while differences among more than two groups were analyzed using analysis of variance. Associations between SALL4 expression and the clinicopathological characteristics in glioma patients were assessed using the $\chi^{2}$ test. Statistical analysis was performed using SPSS version 19.0 (IBM Corp., Armonk, NY, USA). A $\mathrm{P}$-value that was $<0.05$ was considered to indicate a statistically significant difference.

\section{Results}

miR-219 is downregulated in glioma. In the current study, the expression of miR-219 was examined in a total of 63 glioma tissues and 12 normal brain tissues. The RT-qPCR data revealed that the miR-219 levels were significantly decreased in glioma tissues, when compared with the normal brain tissues (Fig. 1A; $\mathrm{P}<0.001$ ). In addition, high-grade gliomas (WHO grade III-IV) presented significantly lower levels of miR-219 as compared with those in low-grade gliomas (WHO grade I-II) (Fig. 1B; P<0.001). Furthermore, miR-219 was significantly downregulated in several common human glioma cell lines (U87, A172, U373 and U251) compared with the normal astrocytes $(\mathrm{P}<0.001$; Fig. 1C). Therefore, miR-219 is concluded to be downregulated in glioma, and may contribute to glioma progression.

Subsequently, the clinical significance of miR-219 expression in glioma was investigated. Glioma tissues were divided into the high and low miR-219 expression groups, according to the mean value of miR-219 levels that served as the cutoff value. It was observed that low miR-219 expression was significantly associated with an advanced WHO grade and KPS index, but not with the age or sex of patients (Table I). Accordingly, these results suggested that decreased expression of miR-219 is significantly associated with glioma progression in glioma patients. 

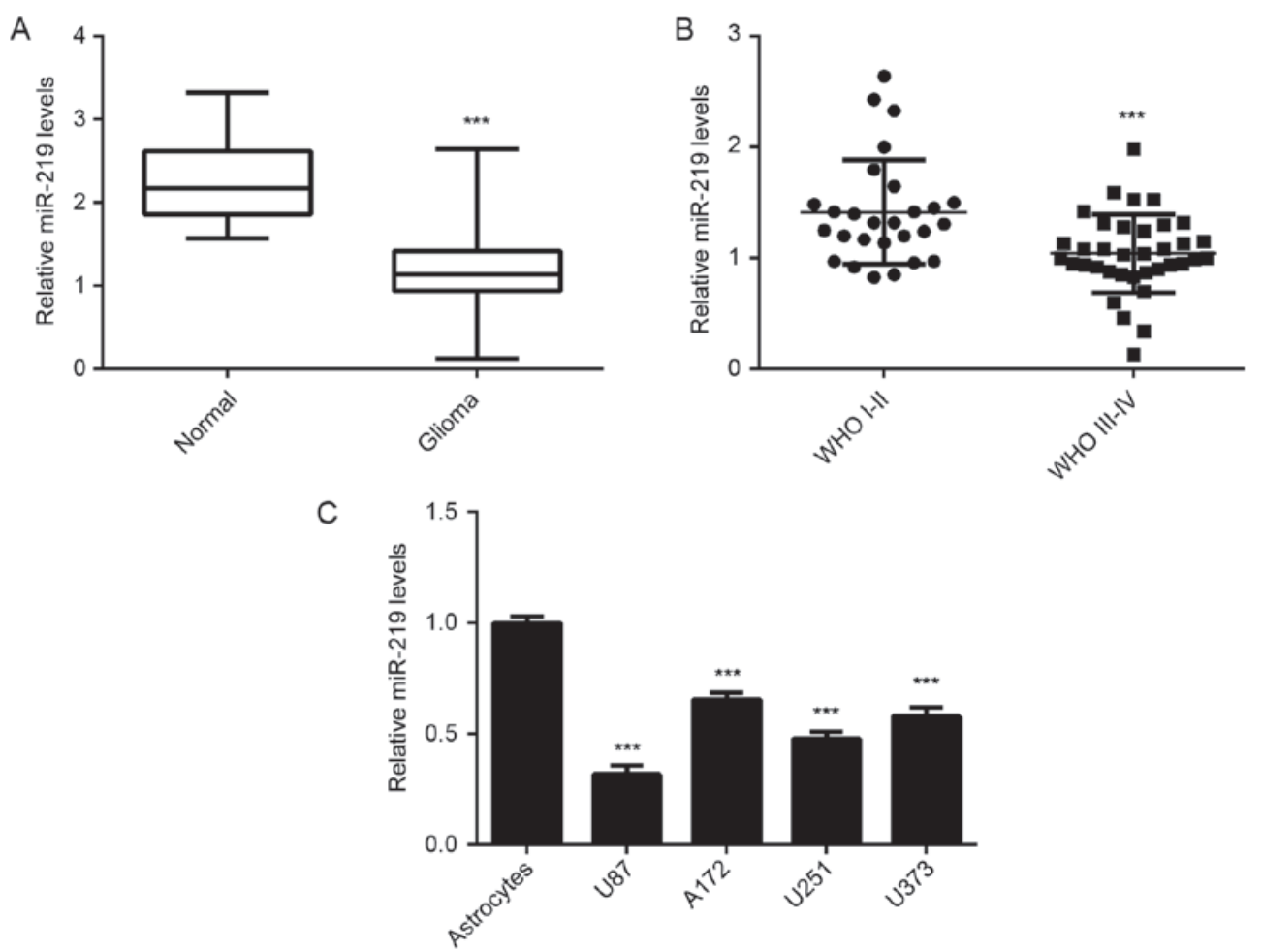

Figure 1. (A) miR-219 expression in 12 normal brain tissues and 63 glioma tissues, examined by RT-qPCR. ${ }^{* * *} \mathrm{P}<0.001$ vs. normal tissues. (B) miR-219 expression in glioma tissues at different WHO grades was determined by RT-qPCR. ${ }^{* * *} \mathrm{P}<0.001$ vs. WHO grade I-II. (C) miR-219 expression in the human glioma cell lines U87, A172, U373 and U251 compared with that in normal astrocytes was determined by RT-qPCR. ${ }^{* * *} \mathrm{P}<0.001$ vs. astrocytes. miR, microRNA; RT-qPCR, reverse transcription-quantitative polymerase chain reaction; WHO, World Health Organization.

A

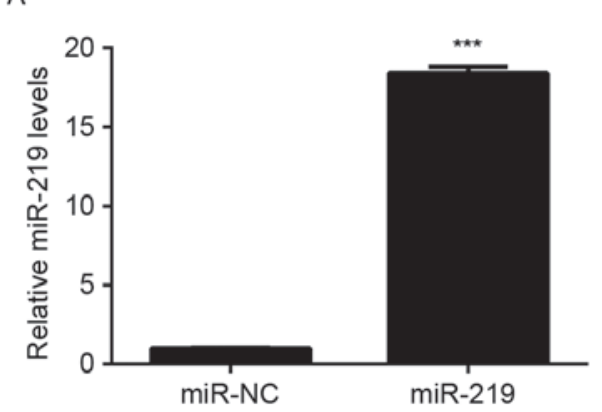

C
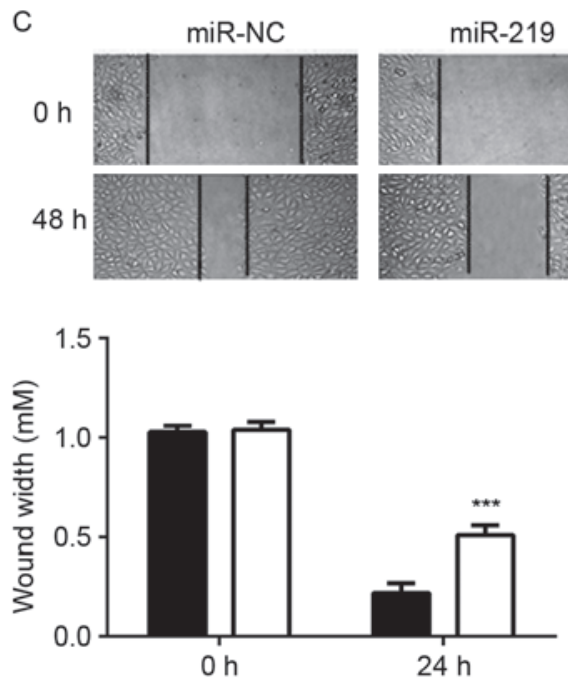

B

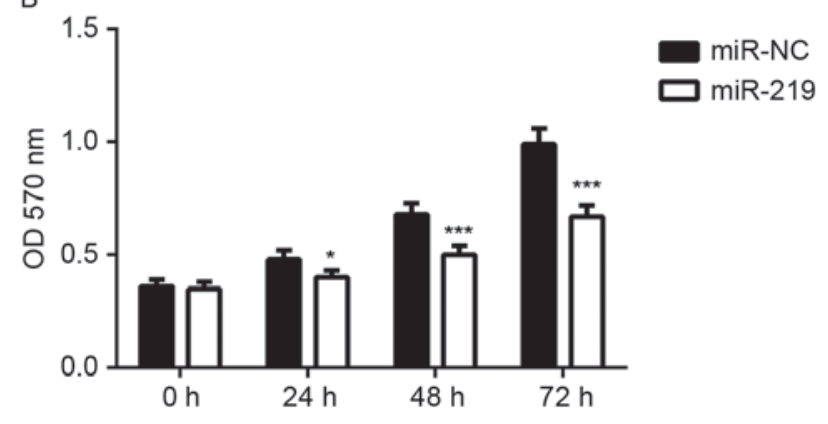

D

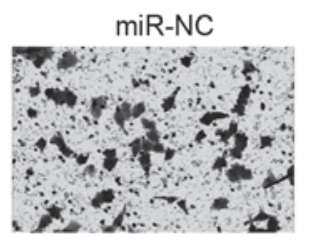

$\operatorname{miR}-219$

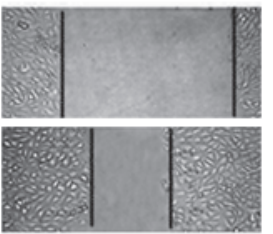

miR-NC

口miR-219

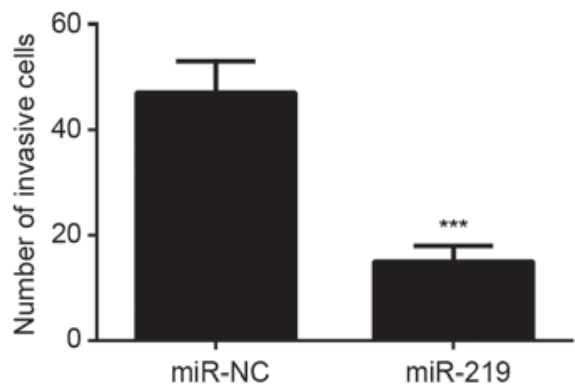

Figure 2. U87 cells were transfected with miR-219 mimic or miR-NC (serving as the control group). (A) Reverse transcription-quantitative polymerase chain reaction was used to examine the miR-219 expression following transfection. (B) MTT, (C) wound healing and (D) transwell assays were conducted to determine the cell proliferation, migration and invasion, respectively. ${ }^{*} \mathrm{P}<0.05$ and ${ }^{* * *} \mathrm{P}<0.001$ vs. miR-NC. miR, microRNA; NC, negative control. 
A

\begin{tabular}{|c|c|c|c|c|c|c|c|}
\hline & $\begin{array}{l}\text { Predicted consequential pairing of target region (top) } \\
\text { and miRNA (bottom) }\end{array}$ & $\begin{array}{l}\text { Site } \\
\text { type }\end{array}$ & $\begin{array}{l}\text { Context++ } \\
\text { score }\end{array}$ & $\begin{array}{l}\text { Context++ score } \\
\text { percentle }\end{array}$ & $\begin{array}{l}\text { Weighted } \\
\text { context++ score }\end{array}$ & $\begin{array}{l}\text { Conserved branch } \\
\text { length }\end{array}$ & $P_{\mathrm{cr}}$ \\
\hline $\begin{array}{l}\text { Position 156-162 of SALL } 43^{\prime} \text { UTR } \\
\text { hsa-mir-219a-5p }\end{array}$ & $\begin{array}{l}5^{5} \text {... CCUCAGGCAAGUGCUACAAUCAC ... } \\
\text { IIIIIII } \\
\text { 3. UCUUAACGCAACCUGUUAGU }\end{array}$ & ${ }_{\mathrm{A} 1}^{7 \mathrm{mer}-}$ & -0.10 & 78 & -0.10 & 6.784 & 0.66 \\
\hline
\end{tabular}

B

Wild type SALL4 3' UTR

5'...AAGUGCUACAAUCA...3'

miR-219

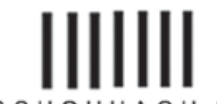

$3^{\prime}$...G C A A A C C U GUUA G U...5'

Mutant type SALL4 3' UTR

$5^{\prime}$...AA G U G C U U G U U A G U...3

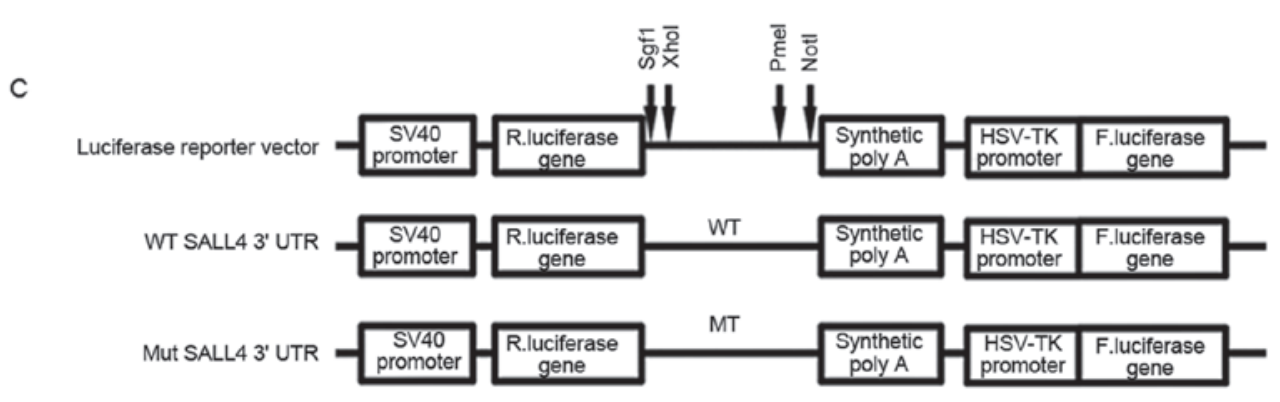

D

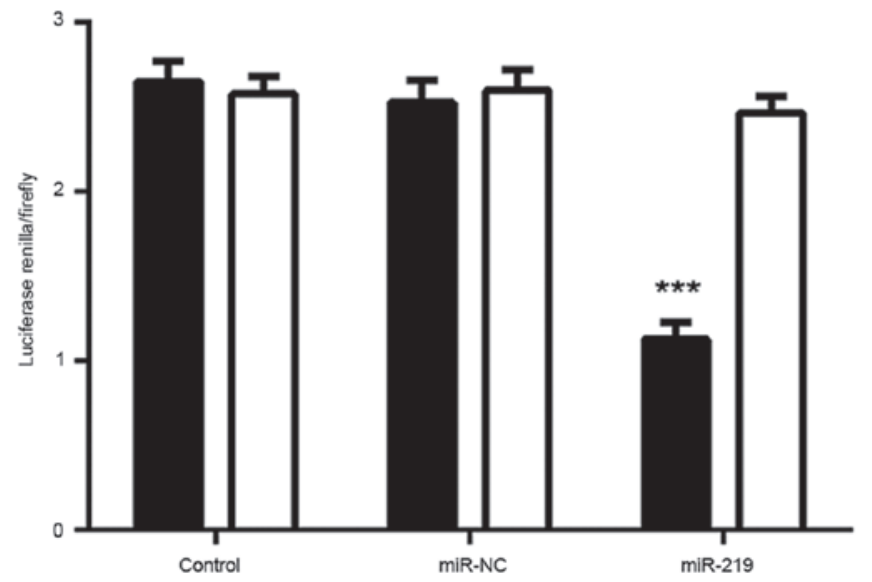

WT SALL4 3'UTR

Mut SALL4 4'UTR

Figure 3. (A) SALL4 is predicted to be a putative target of miR-219. (B) Sequences of WT and Mut SALL4 3'UTR. (C) Luciferase reporter plasmids containing WT or Mut of SALL4 3'UTR were constructed. (D) Luciferase reporter gene assay revealing that the luciferase activity was significantly suppressed in U87 cells co-transfected with the WT-SALL4-3'UTR luciferase reporter plasmid and miR-219 mimic, when compared with the control group, but the luciferase activity was unchanged compared with the control when transfected with Mut-SALL4-3'UTR luciferase reporter plasmid and miR-219 mimic. ${ }^{* * *}$ P $<0.001$ vs. control group. miR, microRNA; WT, wild type; Mut, mutant type; SALL4, Sal-like protein 4; UTR, untranslated region.

Ectopic expression of miR-219 suppresses U87 cell proliferation, migration and invasion. In order to upregulate the expression of miR-219, U87 cells were transfected with miR-219 mimic. Transfection with miR-NC was used as the control group. Following transfection, the miR-219 levels were significantly higher in the miR-219 group compared with those in the miR-NC group (Fig. 2A). MTT, wound healing and transwell assays were then conducted to determine the cell proliferation, migration and invasion, respectively. As indicated in Fig. 2B-D, ectopic expression of miR-219 significantly decreased the proliferation, migration and invasion of U87 cells, when compared with the miR-NC group. These findings suggest that miR-219 may function as a tumor suppressor in glioma.
miR-219 negatively regulates its target gene SALL4 in U87 cells. Next, bioinformatics analysis was performed to predict the potential target genes of miR-219. As shown in Fig. 3A, SALL4 was observed to be a putative target of miR-219. To verify the targeting association between miR-219 and SALL4, luciferase reporter plasmids containing WT or MT SALL4 3'UTRs were constructed (Fig. 3B and C). The results of luciferase reporter gene assay demonstrated that the luciferase activity was significantly inhibited in U87 cells co-transfected with the WT-SALL4-3'UTR luciferase reporter plasmid and miR-219 mimic, when compared with the control group, but the luciferase activity was unchanged compare with the controlwhen transfected with MT-SALL4-3'UTR luciferase reporter plasmid and miR-219 mimic (Fig. 3D). These 
A
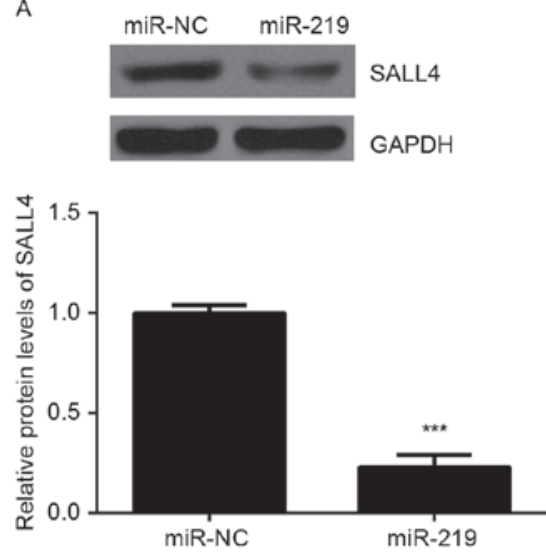

C
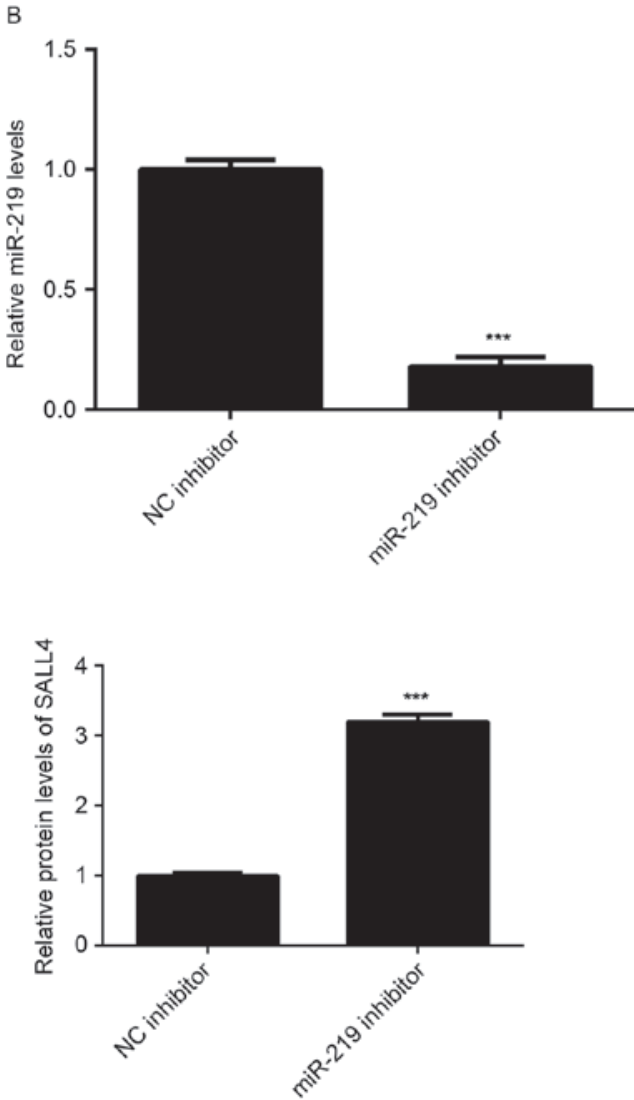

Figure 4. (A) Western blot analysis were used to examine the protein expression of SALL4 in U87 cells transfected with miR-219 mimic or miR-NC. ${ }^{* * *} \mathrm{P}<0.001$ vs. miR-NC group. (B) Reverse transcription-quantitative polymerase chain reaction and (C) western blot analysis were used to examine the miR-219 expression and protein expression of SALL4, respectively, after U87 cells were transfected with miR-219 inhibitor or NC inhibitor. ${ }^{* * *} \mathrm{P}<0.001$ vs. NC inhibitor. miR, microRNA; SALL4, Sal-like protein 4; NC, negative control.

findings indicate that SALL4 is a target gene of miR-219, and that miR-219 directly binds to the 3'UTR of SALL4 mRNA in U87 cells.

As miRs generally inhibit the expression of their target genes at the post-transcriptional level, the present study then investigated the effects of miR-219 on the protein expression of SALL4 in U87 cells. As shown in Fig. 4A, upregulation of miR-219 significantly decreased the protein expression of SALL4. Next, U87 cells were transfected with miR-219 inhibitor to knockdown its expression, with NC inhibitor transfection used as the control group. Following transfection, the miR-219 levels were significantly reduced in the miR-219 inhibitor group, when compared with the NC inhibitor group (Fig. 4B). Western blot analysis data revealed that downregulation of miR-219 significantly increased the protein expression of SALL4 in U87 cells as compared with that in the NC inhibitor group (Fig. 4C). Therefore, these findings suggest that miR-219 negatively regulated the protein expression of its target gene SALL4 in U87 cells.

SALL4 is upregulated in glioma cells. As the earlier experiments observed that miR-219 was downregulated in glioma tissues and that it negatively regulated SALL4 in glioma cells, the expression of SALL4 in glioma tissues and cell lines was subsequently investigated. The results of RT-qPCR indicated that SALL4 was significantly upregulated in glioma tissues as compared with the normal brain tissues (Fig. 5A). Furthermore, the high-grade gliomas (WHO grade III-IV) exhibited higher SALL4 levels in comparison with those in low-grade gliomas (WHO grade I-II; Fig. 5B). Similarly, SALL4 expression levels were also higher in glioma cell lines compared with normal astrocytes (Fig. 5C and D). These observations reveal that the increased expression of SALL4 in glioma may be caused by the downregulation of miR-219.

Overexpression of SALL4 attenuated the suppressive effects of miR-219 on the malignant phenotypes of U87 cells. The current study further examined whether SALL4 was involved in the miR-219-mediated proliferation, migration and invasion of U87 cells. miR-219-overexpressing U87 cells were transfected with SALL4 expression plasmid, in order to upregulate its expression. Following transfection, the mRNA and protein levels of SALL4 were significantly higher in the miR-219 + SALL4 group compared with those in the miR-219 group (Fig. 6A and B). MTT, wound healing and transwell assays were subsequently conducted to determine cell proliferation, migration and invasion, respectively. As indicated in Fig. 6C-E, the proliferation, migration and invasion of U87 cells were significantly upregulated in the miR-219 + SALL4 group when compared with those in the miR-219 group. Accordingly, overexpression of SALL4 attenuated the suppressive effects of miR-219 upregulation on the malignant phenotypes of U87 cells, suggesting that miR-219 serves a suppressive role in glioma cells via directly targeting SALL4. 


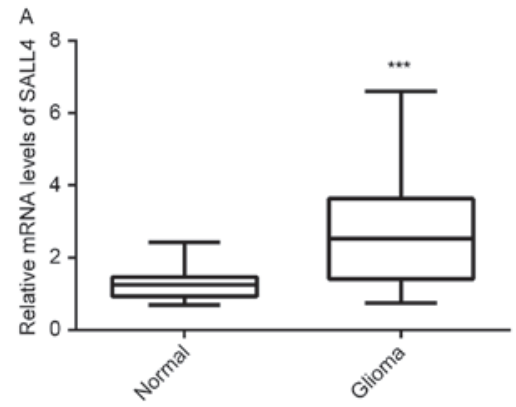

B
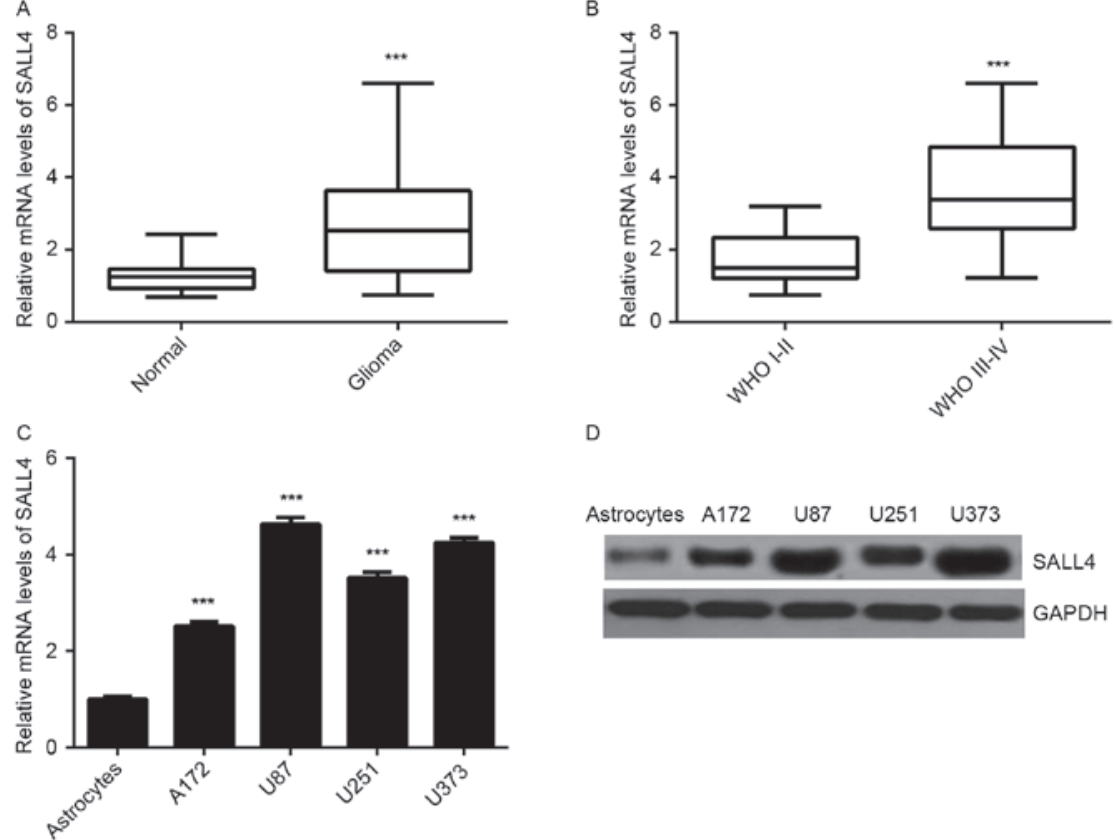

$\mathrm{D}$

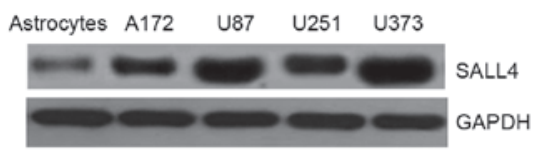

Figure 5. (A) RT-qPCR was used to examine the mRNA expression of SALL4 in 12 normal brain tissues and 63 glioma tissues. ${ }^{* * *} \mathrm{P}<0.001$ vs. normal tissues. (B) RT-PCR was used to examine the mRNA expression of SALL4 in glioma tissues at different WHO grades. ${ }^{* * *}$ P $<0.001$ vs. WHO grade I-II. (C) RT-qPCR and (D) western blot analysis were used to examine the mRNA and protein expression levels of SALL4 in human glioma cell lines U87, A172, U373 and U251 as compared with normal astrocytes. ${ }^{* * *} \mathrm{P}<0.001$ vs. astrocytes. RT-qPCR, reverse transcription-quantitative polymerase chain reaction; WHO, World Health Organization; SALL4, Sal-like protein 4.
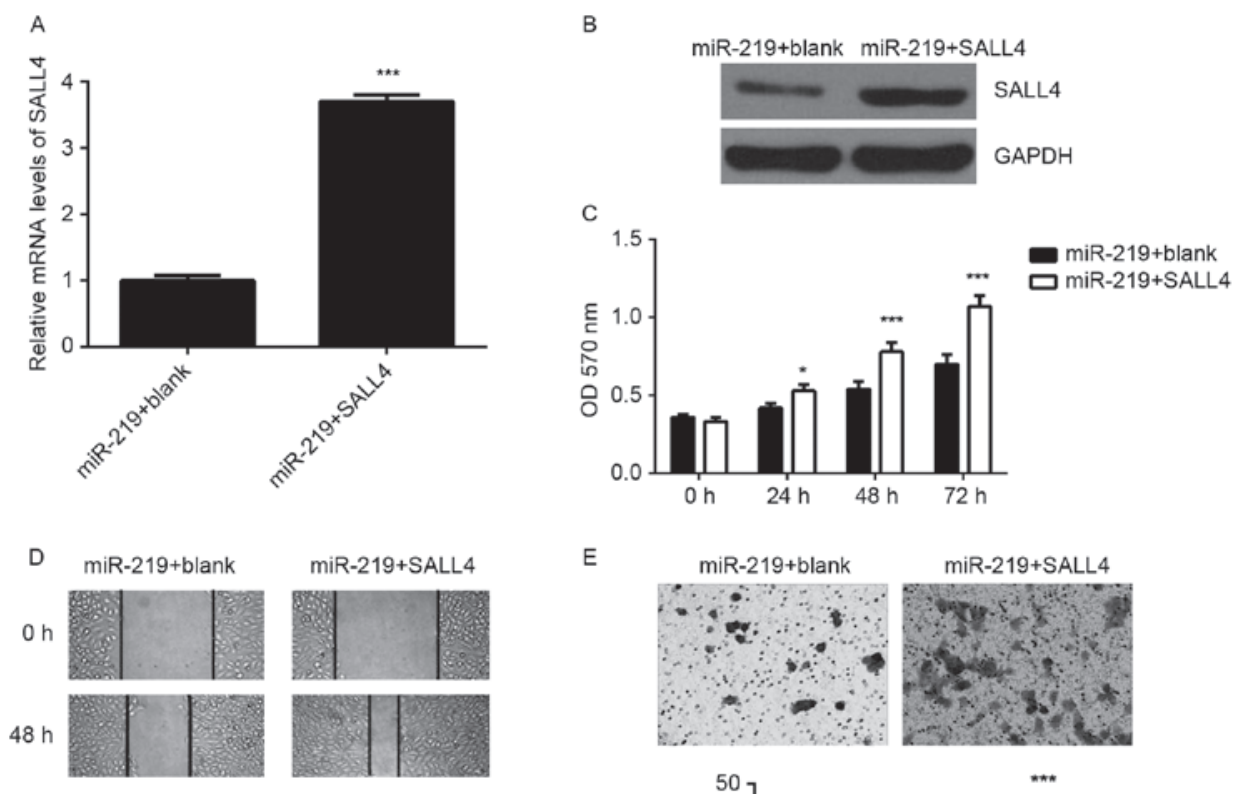

E
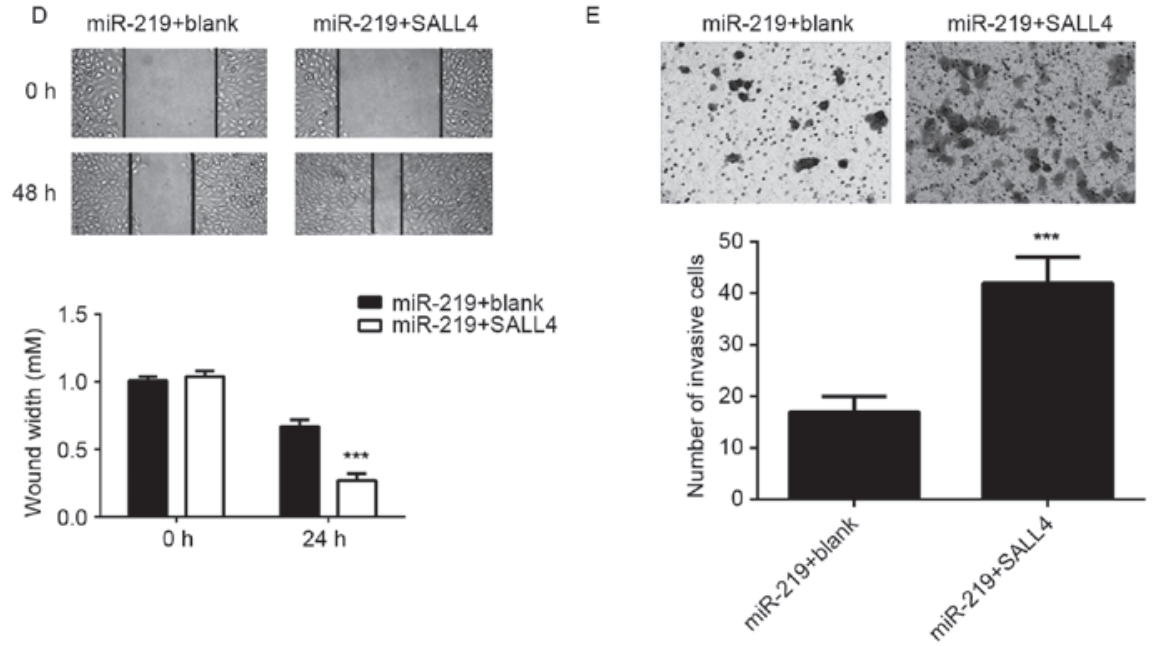

Figure 6. miR-219-overexpressing U87 cells were transfected with SALL4 expression plasmid or blank vector. (A) Reverse transcription-quantitative polymerase chain reaction and (B) western blot analysis were used to examine the mRNA and protein expression levels of SALL4, respectively. (C) MTT, (D) wound healing and (E) transwell assays were conducted to determine the cell proliferation, migration and invasion, respectively. ${ }^{*} \mathrm{P}<0.05$ and ${ }^{* * * *} \mathrm{P}<0.001$ vs. miR-219 + blank group. miR, microRNA; SALL4, Sal-like protein 4. 


\section{Discussion}

MiR-219 was recently suggested to function as a tumor suppressor in glioma, however, the underlying mechanism remains largely unknown. In the present study, it was observed that miR-219 was significantly downregulated in glioma tissues and cell lines, and that reduced expression of miR-219 was significantly associated with advanced pathological grade in glioma tissues. Furthermore, ectopic expression of miR-219 inhibited the proliferation, migration and invasion of U87 cells. SALL4, which was markedly upregulated in glioma, was further identified as a target gene of miR-219 in U87 cells, while overexpression of SALL4 significantly eliminated the suppressive effects of miR-219 on U87 cell proliferation, migration and invasion.

Recently, miR-219 has been reported to serve a suppressive role in malignant tumors in the central nervous system (17). For instance, Shi et al reported that miR-219 inhibited the proliferation, migration and invasion of medulloblastoma cells by targeting CD164 (17). In the present study, it was observed that miR-219 was downregulated in glioma tissues compared with normal brain tissues, as well as in glioma cell lines as compared with normal astrocytes. The findings of the present study are consistent with a previous study (12). In addition, low expression of miR-219 was identified to be significantly associated with high tumor grade, suggesting that downregulation of miR-219 may contribute to the malignant progression of glioma. Furthermore, it was demonstrated that ectopic expression of miR-219 decreased the proliferation, migration and invasion of U87 cells. Consistent with the present study data, Jiang et al (12) also reported that miR-219 inhibited cell proliferation and invasion, induced the apoptosis of glioblastoma cells in vitro, and inhibited xenograft formation in vivo. Rao et al (13) demonstrated that overexpression of miR-219 in glioma cell lines inhibited the proliferation, growth and migration via inhibition of receptor tyrosine kinase pathway through directly targeting EGFR. Rao et al (18) also reported that overexpression of miR-219-5p caused decreased soft agar colony formation of glioblastoma cells.

The present study further identified SALL4 as a target gene of miR-219, and the protein levels of SALL4 were negatively mediated by miR-219 in U87 cells. SALL4, a zinc finger transcription factor, is considered as an important marker for stem cells and participates in the maintenance of embryonic stem cell self-renewal (19). Recently, SALL4 has been demonstrated to be significantly upregulated in certain types of human cancer, including hepatocellular carcinoma (20), intrahepatic cholangiocarcinoma (21), esophageal squamous cell carcinoma (22), gastric cancer (23) and lung cancer (24). For instance, Deng et al (21) showed that the increased expression of SALL4 contributed to the malignant progression in intrahepatic cholangiocarcinoma, as well as to the poor prognosis of patients. It has been demonstrated that SALL4 can promote tumor cell growth, metastasis and angiogenesis, as well as induce drug resistance (21-23). Oikawa et al (25) reported that overexpression of SALL4 increased hepatocellular carcinoma cell proliferation in vitro, while knockdown of SALL4 led to a significant decrease in growth inhibition in vitro and in vivo. Kobayashi et al (24) observed that inhibition of SALL4 inhibited the proliferation of lung cancer cells through induction of a cell cycle arrest at the G1 phase. In the present study, SALL4 was significantly upregulated in glioma tissues and cell lines, while high-grade gliomas (WHO grade III-IV) presented higher SALL4 levels when compared with those in low-grade tumors (WHO grade I-II). These findings are consistent with a previous study reporting that increased expression of SALL4 was associated with advanced pathological grade in glioma (26). Therefore, the increased expression of SALL4 may be caused by the reduced miR-219 levels in glioma tissues.

As SALL4 was negatively regulated by miR-219 in U87 cells, SALL4 may be involved in the miR-219-mediated malignant phenotypes of U87 cells. To verify this hypothesis, miR-219-overexpressing U87 cells were then transfected with SALL4 expression plasmid to upregulate its expression. The results in the current study indicated that overexpression of SALL4 significantly attenuated the inhibitory effects of miR-219 upregulation on U87 cell proliferation, migration and invasion. These findings support that the tumor suppressive role of miR-219 in glioma cells is, at least partly, via inhibition of SALL4 expression. Similarly, a previous study reported that miR-107 inhibited glioma cell proliferation and induced cell apoptosis via directly targeting SALL4 (27). Therefore, the present study further highlights the importance of the $\mathrm{miR} / \mathrm{SALL} 4$ axis in glioma.

In conclusion, to the best of our knowledge, the current study is the first to demonstrate that miR-219 exerts suppressive effects on the malignant phenotypes of glioma cells, which was, at least partly, through inhibition of SALL4 expression. These findings suggest that the miR-219/SALL4 association may be a potential therapeutic target for glioma.

\section{References}

1. Torre LA, Bray F, Siegel RL, Ferlay J, Lortet-Tieulent J and Jemal A: Global cancer statistics, 2012. CA Cancer J Clin 65: 87-108, 2015.

2. Siegel RL, Miller KD and Jemal A: Cancer statistics, 2015. CA Cancer J Clin 65: 5-29, 2015.

3. Marumoto T and Saya H: Molecular biology of glioma. Adv Exp Med Biol 746: 2-11, 2012.

4. Ambros V: The functions of animal microRNAs. Nature 431: 350-355, 2004.

5. Bartel DP: MicroRNAs: Genomics, biogenesis, mechanism, and function. Cell 116: 281-297, 2004.

6. Calin GA, Sevignani C, Dumitru CD, Hyslop T, Noch E, Yendamuri S, Shimizu M, Rattan S, Bullrich F, Negrini M and Croce CM: Human microRNA genes are frequently located at fragile sites and genomic regions involved in cancers. Proc Natl Acad Sci USA 101: 2999-3004, 2004.

7. Croce CM and Calin GA: miRNAs, cancer, and stem cell division. Cell 122: 6-7, 2005.

8. An L, Liu Y, Wu A and Guan Y: microRNA-124 inhibits migration and invasion by down-regulating ROCK1 in glioma. PLoS One 8: e69478, 2013.

9. Jia Z, Wang K, Wang G, Zhang A and Pu P: MiR-30a-5p antisense oligonucleotide suppresses glioma cell growth by targeting SEPT7. PLoS One 8: e55008, 2013.

10. Lei H, Zou D, Li Z, Luo M, Dong L, Wang B, Yin H, Ma Y, Liu C, Wang F, et al: MicroRNA-219-2-3p functions as a tumor suppressor in gastric cancer and is regulated by DNA methylation. PLoS One 8: e60369, 2013.

11. Huang N,Lin J, Ruan J,Su N, Qing R,Liu F,He B,Lv C,Zheng D and Luo R: MiR-219-5p inhibits hepatocellular carcinoma cell proliferation by targeting glypican-3. FEBS Lett 586: 884-891, 2012.

12. Jiang $Y$, Yin L, Jing $H$ and Zhang H: MicroRNA-219-5p exerts tumor suppressor function by targeting ROBO1 in glioblastoma. Tumour Biol 36: 8943-8951, 2015. 
13. Rao SA, Arimappamagan A, Pandey P, Santosh V, Hegde AS, Chandramouli BA and Somasundaram K: miR-219-5p inhibits receptor tyrosine kinase pathway by targeting EGFR in glioblastoma. PLoS One 8: e63164, 2013.

14. Komori T: The 2016 WHO classification of tumours of the central nervous system: The major points of revision. Neurol Med Chir (Tokyo) 57: 301-311, 2017.

15. Terret C, Albrand G, Moncenix G and Droz JP: Karnofsky performance scale (KPS) or physical performance test (PPT)? That is the question. Crit Rev Oncol Hematol 77: 142-147, 2011.

16. Livak KJ and Schmittgen TD: Analysis of relative gene expression data using real-time quantitative PCR and the 2(-Delta Delta C(T)) method. Methods 25: 402-408, 2001.

17. Shi JA, Lu DL, Huang $X$ and Tan W: miR-219 inhibits the proliferation, migration and invasion of medulloblastoma cells by targeting CD164. Int J Mol Med 34: 237-243, 2014.

18. Rao SA, Santosh V and Somasundaram K: Genome-wide expression profiling identifies deregulated miRNAs in malignant astrocytoma. Mod Pathol 23: 1404-1417, 2010.

19. Chen X, Vega VB and Ng HH: Transcriptional regulatory networks in embryonic stem cells. Cold Spring Harb Symp Quant Biol 73: 203-209, 2008

20. Han SX, Wang JL, Guo XJ, He CC, Ying X, Ma JL, Zhang YY, Zhao Q and Zhu Q: Serum SALL4 is a novel prognosis biomarker with tumor recurrence and poor survival of patients in hepatocellular carcinoma. J Immunol Res 2014: $262385,2014$.
21. Deng G, Zhu L, Huang F, Nie W, Huang W, Xu H, Zheng S, Yi Z and Wan T: SALL4 is a novel therapeutic target in intrahepatic cholangiocarcinoma. Oncotarget 6: 27416-27426, 2015.

22. Forghanifard MM, Ardalan Khales S, Javdani-Mallak A, Rad A, Farshchian M and Abbaszadegan MR: Stemness state regulators SALL4 and SOX2 are involved in progression and invasiveness of esophageal squamous cell carcinoma. Med Oncol 31: 922, 2014.

23. Zhang L, Xu Z, Xu X, Zhang B, Wu H, Wang M, Zhang X, Yang T, Cai J, Yan Y, et al: SALL4, a novel marker for human gastric carcinogenesis and metastasis. Oncogene 33: 5491-5500, 2014.

24. Kobayashi D, Kuribayashi K, Tanaka M and Watanabe N: Overexpression of SALL4 in lung cancer and its importance in cell proliferation. Oncol Rep 26: 965-970, 2011.

25. Oikawa T, Kamiya A, Zeniya M, Chikada H, Hyuck AD, Yamazaki Y, Wauthier E, Tajiri H, Miller LD, Wang XW, et al: Sal-like protein 4 (SALL4), a stem cell biomarker in liver cancers. Hepatology 57: 1469-1483, 2013.

26. Zhang L, Yan Y, Jiang Y, Cui Y, Zou Y, Qian J, Luo C, Lu Y and $\mathrm{Wu} X$ : The expression of SALL4 in patients with gliomas: High level of SALL 4 expression is correlated with poor outcome. J Neurooncol 121: 261-268, 2015.

27. He J, Zhang W, Zhou Q, Zhao T, Song Y, Chai L and Li Y: Low-expression of microRNA-107 inhibits cell apoptosis in glioma by upregulation of SALL4. Int J Biochem Cell Biol 45: 1962-1973, 2013. 